\title{
JENSEN'S INEQUALITY FOR POSITIVE CONTRACTIONS ON OPERATOR ALGEBRAS
}

\author{
DÉNES PETZ
}

\begin{abstract}
Let $\tau$ be a normal semifinite trace on a von Neumann algebra, and let $f$ be a continuous convex function on the interval $[0, \infty)$ with $f(0)=0$. For a positive element $a$ of the algebra and a positive contraction $\alpha$ on the algebra, the following inequality is obtained:
\end{abstract}

$$
\tau(f(\alpha(a))) \leq \tau(\alpha(f(a))) .
$$

Versions of Jensen's inequality play in important role in several parts of mathematics and applications. One of the standard tools in quantum statistical mechanics is the Peierls-Bogoliubov inequality

$$
\operatorname{tr} f(A) \geq \sum_{i} f\left(\left\langle A \eta_{i}, \eta_{i}\right\rangle\right)
$$

where $f$ is a convex function on the real line, $A$ is a selfadjoint operator, and $\left\{\eta_{i}\right\}$ is an orthonormal set (see [11], for example). Slightly more general, if $\left\{P_{i}\right\}$ is a pairwise orthogonal family of projections and we set $\alpha(A)=\sum_{i} P_{i} A P_{i}$ for a selfadjoint operator $A$, then

$$
\operatorname{tr} \alpha(f(A)) \geq \operatorname{tr} f(\alpha(A))
$$

(see [2, 6, and 9]). Going further in this direction Brown and Kosaki [3] have recently proved that

$$
\tau\left(f\left(v^{*} a v\right)\right) \leq \tau\left(v^{*} f(a) v\right),
$$

where $\tau$ is a faithful normal semifinite trace on a von Neumann algebra, $v$ is a contraction, and $a$ is a positive element of the algebra. (In fact, $a$ may be unbounded and affiliated with the algebra.) In the present paper we obtain a similar inequality for an arbitrary positive contraction $\alpha$ on the algebra and show that

$$
\tau(f(\alpha(a))) \leq \tau(\alpha(f(a))) .
$$

Here $f$ is supposed to be a continuous convex function with $f(0)=0$. (Note that $(*)$ holds without $\tau$ if $f$ is operator convex; cf. Theorem IV.1 in [1].) The inequality $(*)$ supports the experience that inequalities involving operators are more simply treatable provided both sides are inside of a trace (see [3 and 8] for further evidences).

The idea of our proof is to approximate the operators $a$ and $\alpha(a)$ by diagonal ones by means of their spectral resolution and to apply the classical Jensen's inequality in

Received by the editors November 19, 1984 and, in revised form, June 24, 1985 and December 17, 1985.

1980 Mathematics Subject Classification (1985 Revision). Primary 46L50; Secondary 26A51, 47B15.

Key words and phrases. Convex function, trace, von Neumann algebra, Jensen's inequality. 
its discrete form. Comparing with $[\mathbf{3}]$ this way is more direct. (The main technical tool in $[\mathbf{3}]$ is the spectral dominance which is intimately related to the generalized $s$-numbers. See [3 Remark 5; 5, and 8].)

Our basic references on operator algebras are $[\mathbf{4}$ and 10]. We recall that if $\tau$ is a normal semifinite trace on a von Neumann algebra $M$, then $\tau$ is weak* lower semicontinuous and admits an extension to a linear functional on the ideal linearly spanned by the set $\left\{a \in M_{+}: \tau(a)<+\infty\right\}$ (see [4, Chapter I, §6]. Let $h$ be a selfadjoint operator with Jordan decomposition $h_{1}-h_{2}$. Following [3] we say that $\tau(h)$ is defined if $\tau\left(h_{1}\right)<+\infty$ or $\tau\left(h_{2}\right)<+\infty$. In this case, we set $\tau(h)=\tau\left(h_{1}\right)-\tau\left(h_{2}\right)$. If both $\tau(h)$ and $\tau\left(h^{\prime}\right)$ are defined and $\tau(h)+\tau\left(h^{\prime}\right)$ is well defined (i.e., does not have the form $\infty-\infty)$, then $\tau\left(h+h^{\prime}\right)=\tau(h)+\tau\left(h^{\prime}\right)$ (cf. Lemma 9 in $[3]$ ).

THEOREM A. Let $M$ and $N$ be von Neumann algebras and $f: \mathbf{R}^{+} \rightarrow \mathbf{R}$ be a continuous convex function with $f(0)=0$. Assume that $\tau$ is a normal semifinite trace on $M$ and $\alpha: N \rightarrow M$ is a positive contraction. Then for every $a \in N_{+}$the inequality

$$
\tau(f(\alpha(a))) \leq \tau(\alpha(f(a)))
$$

holds provided that both sides are defined.

PROOF. First we show that

$$
\tau(p f(p \alpha(a) p) p) \leq \tau(p \alpha(f(a)) p)
$$

for any projection $p$ in $M$ with $\tau(p)<+\infty$ and for any $a \in N_{+}$. (Note that in this case both sides of (1) are defined and finite.) Take an arbitrary $\varepsilon>0$. We approximate $p \alpha(a) p$ by a diagonal operator $\sum_{i=1}^{n} \mu_{i} p_{i}$ such that $p_{i}$ is a spectral projection of $p \alpha(a) p$ for $1 \leq i \leq n, \sum_{i=1}^{n} p_{i}=p$,

$$
\tau(p f(p \alpha(a) p) p) \leq \sum_{i=1}^{n} f\left(\mu_{i}\right) \tau\left(p_{i}\right)+\varepsilon,
$$

and

$$
\mu_{i}=\left(\begin{array}{ll}
\tau\left(\alpha(a) p_{i}\right) / \tau\left(p_{i}\right) & \text { when } \tau\left(p_{i}\right)>0, \\
0 & \text { otherwise. }
\end{array}\right.
$$

We choose $\delta>0$ such that $\left|t_{1}-t_{2}\right| \leq \delta$ and $0 \leq t_{1} \leq\|a\|$ imply $\left|f\left(t_{1}\right)-f\left(t_{2}\right)\right| \leq$ $\varepsilon / \tau(p)$. There exists a diagonal operator $b=\sum_{j=1}^{k} \nu_{j} q_{j}$ such that $\|a-b\| \leq \delta$, $\sum_{j=1}^{k} q_{j}=I$, and

$$
|\tau(p \alpha(f(a)) p)-\tau(p \alpha(f(b)) p)| \leq \varepsilon .
$$

By simple calculation we have

$$
\begin{aligned}
f\left(\mu_{i}\right) & =f\left(\sum_{j=1}^{k} \tau\left(\alpha\left(a q_{j}\right) p_{i}\right) / \tau\left(p_{i}\right)\right) \\
& =f\left(\sum_{j=1}^{k} \nu_{j} \tau\left(\alpha\left(q_{j}\right) p_{i}\right) / \tau\left(p_{i}\right)+\tau\left(\alpha(a-b) p_{i}\right) / \tau\left(p_{i}\right)\right)
\end{aligned}
$$


when $\tau\left(p_{i}\right)>0$ and $1 \leq i \leq n$. Here

$$
\left|\tau\left(\alpha(a-b) p_{i}\right) / \tau\left(p_{i}\right)\right| \leq\|\alpha(a-b)\| \leq \delta
$$

and so

$$
f\left(\mu_{i}\right) \leq f\left(\sum_{j=1}^{k} \nu_{j} \tau\left(\alpha\left(q_{j}\right) p_{i}\right) / \tau\left(p_{i}\right)\right)+\varepsilon / \tau(p) .
$$

Now apply Jensen's inequality

$$
f\left(\mu_{i}\right) \leq \sum_{j=1}^{k} f\left(\nu_{j}\right) \tau\left(\alpha\left(q_{j}\right) p_{i}\right) / \tau\left(p_{i}\right)+\varepsilon / \tau(p) .
$$

Consequently

$$
\begin{aligned}
\sum_{i=1}^{n} f\left(\mu_{i}\right) \tau\left(p_{i}\right) & \leq \sum_{j=1}^{k} f\left(\nu_{j}\right) \tau\left(\alpha\left(q_{j}\right)\left(\sum_{i=1}^{n} p_{i}\right)\right)+\varepsilon \\
& =\tau(p \alpha(f(b)) p)+\varepsilon .
\end{aligned}
$$

Finally we arrive at the estimate

$$
\tau(p f(p \alpha(a) p) p) \leq \tau(p \alpha(f(a)) p)+3 \varepsilon
$$

and since $\varepsilon$ was arbitrary (1) is proved.

When $\tau$ is finite the proof is complete. If not, then a supplementary argument is needed. There is $c \in \mathbf{R}^{+} \cup\{+\infty\}$ such that $f \geq 0$ on $(c,+\infty)=I_{1}$ and $f \leq 0$ on $[0, c]=I_{2}$. Let $q_{i}$ be the spectral projection of $\alpha(a)$ corresponding to $I_{i}(i=1,2)$. Since $q_{1} f(\alpha(a))-q_{2}(-f(\alpha(a)))$ is the Jordan decomposition of $f(\alpha(a))$ we have

$$
\tau(f(\alpha(a)))=\tau\left(q_{1} f(\alpha(a))\right)+\tau\left(q_{2} f(\alpha(a))\right) .
$$

Let $d_{1}-d_{2}$ be the Jordan decomposition of $\alpha(f(a))$. Since $\tau\left(d_{1}\right)<\infty$ or $\tau\left(d_{2}\right)<\infty$ the difference $\tau\left(p d_{1}\right)-\tau\left(p d_{2}\right)$ is well defined and equal to $\tau(p \alpha(f(a)))$ for any projection $p$.

By the semifiniteness of $\tau$ there exists an increasing net $\left(p_{s}^{i}\right)_{s \in S}$ of projections in $M$ such that $p_{s}^{i} \rightarrow q_{i}(i=1,2)$ and $\tau\left(I p_{s}^{i}\right)<+\infty(i=1,2$ and $s \in S)$. For any $s \in S$ we have proved that

$$
\tau\left(p_{s}^{i} f\left(p_{s}^{i} \alpha(a) p_{s}^{i}\right) p_{s}^{i}\right) \leq \tau\left(p_{s}^{i} \alpha(f(a)) p_{s}^{i}\right)
$$

Since $\tau$ is weak* lower semicontinuous and

$$
p_{s}^{i} f\left(p_{s}^{i} \alpha(a) p_{s}^{i}\right) p_{s}^{i} \rightarrow q_{i} f\left(q_{i} \alpha(a) q_{i}\right)=q_{i} f(\alpha(a))
$$

in the strong operator topology $[10$, II, 4.6] and hence in the weak* topology, we establish

$$
\begin{aligned}
\tau\left(q_{i} f(\alpha(a))\right) & \leq \liminf _{s} \tau\left(p_{s}^{i} f\left(p_{s}^{i} \alpha(a) p_{s}^{i}\right) p_{s}^{i}\right) \\
& \leq \lim _{s} \tau\left(p_{s}^{i} d_{1}\right)-\lim _{s} \tau\left(p_{s}^{i} d_{2}\right)=\tau\left(q_{i} \alpha(f(a))\right)
\end{aligned}
$$

Adding these inequalities for $i=1$ and $i=2$ we complete the proof. 
In the whole proof of the theorem we needed $f(0)=0$ only when the discrete Jensen's inequality was applied to a degenerate convex combination

$$
\sum_{j=1}^{k} \nu_{j} \tau\left(\alpha\left(q_{j}\right) p_{i}\right) / \tau\left(p_{i}\right)
$$

(that is, $\sum_{j=1}^{k} \tau\left(\alpha\left(q_{j}\right) p_{i}\right) / \tau\left(p_{i}\right) \leq 1$ ). If we replace $f(0)=0$ by the assumption that $\alpha$ is unit preserving, then

$$
\sum_{j=1}^{k} \tau\left(\alpha\left(q_{j}\right) p_{i}\right) / \tau\left(p_{i}\right)=\tau\left(\alpha\left(\sum_{j=1}^{k} q_{j}\right) p_{i}\right) / \tau\left(p_{i}\right)=1
$$

and the same arguments give another version of the previous theorem. (We recall that a positive unit preserving linear mapping is necessarily a contraction; see Lemma 1 in [7], for example.)

THEOREM B. Let $M$ and $N$ be von Neumann algebras and $f: \mathbf{R}^{+} \rightarrow \mathbf{R}$ be $a$ continuous convex function with $f(0)=0$. Assume that $\tau$ is a normal semifinite trace on $M$ and $\alpha: N \rightarrow M$ is a positive unit preserving mapping. Then for every $a \in N_{+}$the inequality

$$
\tau(f(\alpha(a))) \leq \tau(\alpha(f(a)))
$$

holds whenever both sides are defined.

Theorems $\mathrm{A}$ and $\mathrm{B}$ have a simple consequence for positive contractions on $C^{*}$ algebras. Recall that a positive functional $\tau$ on a $C^{*}$-algebra is called tracial if $\tau\left(a a^{*}\right)=\tau\left(a^{*} a\right)$ for every element $a$.

Corollary. Let $A$ and $B$ be unitial $C^{*}$-algebras and $\alpha: A \rightarrow B$ be a positive contraction. Assume that $f: \mathbf{R}^{+} \rightarrow \mathbf{R}$ is a continuous convex function. If $\tau$ is a tracial positive functional on $B$ and

(i) $\alpha$ is unit preserving, or

(ii) $f(0)=0$,

then the inequality (*) holds for every positive $a \in A$.

PROOF. The second adjoint of $\alpha$ is an extension of $\alpha$ and forms a positive contraction of $A^{* *}$ into $B^{* *}$. On the other hand, $\tau$ admits an extension to a normal tracial positive functional of the enveloping von Neumann algebra $B^{* *}$. So one simply applies Theorems $\mathrm{A}$ and $\mathrm{B}$ for the enveloping von Neumann algebras $A^{* *}$ and $B^{* *}$.

This paper was completed while the author stayed at the Mathematical Institute of the University of Tübingen as an Alexander von Humboldt-Fellow.

\section{REFERENCES}

1. T. Ando, Topics on operator inequalities, Lecture Notes, Hokkaido University, Sapporo, 1978.

2. F. A. Berezin, Convex operator functions, Math. Sb. 88 (1972), 268-276. (Russian)

3. L. G. Brown and H. Kosaki, Jensen's inequality in semifinite von Neumann algebras, J. Operator Theory (to appear).

4. J. Dixmier, Les algèbres d'opérateurs dans l'espace Hilbertien (Algébres de von Neumann), 2nd ed., Gauthier-Villars, Paris, 1969. 
5. T. Fack, Sur la notion de valeur caractéristique, J. Operator Theory 7 (1982), 307-330.

6. G. Lindblad, Expectations and entropy inequalities for finite quantum systems, Comm. Math. Phys. 39 (1974), 111-119.

7. D. Petz, A dual in von Neumann algebras with weights, Quart. J. Math. Oxford Ser. 35 (1984), 475-483.

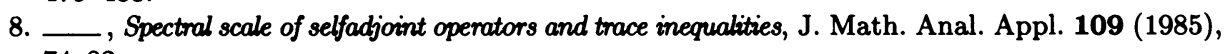
74-82.

9. B. Simon, Trace ideals and their applications, Cambridge Univ. Press, Cambridge, 1979.

10. M. Takesaki, Operator algebras. I, Springer-Verlag, Berlin and New York, 1979.

11. A. Wehrl, General properties of entropy, Rev. Modern Phys. 50 (1978), 221-260.

MATHEMATICS INSTITUTE OF THE HUNGARIAN ACADEMY OF SCIENCES, REÁltANODA U. 13-15, 1364 BUDAPEST, PF. 127, HUNGARY 